\title{
Has the COVID-19 Pandemic Increased Mortality Among Patients with Cancer Receiving Systemic Anticancer Treatments?
}

\author{
Ayşe Demirci ${ }^{1}$, Cemil Bilir ${ }^{1}$, Emre Cakir ${ }^{1}$, Burcu Gulbagci ${ }^{1}$, Ilhan Hacibekiroglu ${ }^{1}$ and Ceyhun Varim ${ }^{2}$ \\ ${ }^{1}$ Department of Medical Oncology, Sakarya University Training and Research Hospital, Sakarya, Turkey \\ ${ }^{2}$ Department of Internal Medicine, Sakarya University Training and Research Hospital, Sakarya, Turkey
}

\begin{abstract}
Objective: To evaluate the mortality rates in patients receiving anticancer therapy in the coronavirus disease-19 (COVID-19) pandemic period.

Study Design: Descriptive study.

Place and Duration of Study: Department of Medical Oncology, Sakarya University Training and Research Hospital, Sakarya, Turkey, from December 2017 to May 2020.

Methodology: Only patients who received chemotherapy and immunotherapy were selected and enrolled in the study. All patients $(n=3,204)$ were divided into three groups, namely the first group (1st December 2017-31st May 2018, $n=918$ ), second group (1st December 2018-31st May 2019, $n=1,147$ ), and the pandemic period group (PPG) (1st December 2019-31st May $2020, n=1,139$ ), according to the period during which they received anticancer treatment. The clinical and demographic characteristics and mortality rates of these three groups of patients were compared.

Results: The median age of the total of 3,204 patients was 61 (53-69). In this study, $51.1 \%(n=1,636)$ were females and $48.9 \%$ were males. The mortality rates were $13.5 \%(n=124)$ in the first group, $13.4 \%(n=154)$ in the second group, and $13.0 \%(n=148)$ in the PPG, respectively. Overall mortality rates did not differ among patients with cancer in the three different six-month periods analysed $(p=0.931)$.

Conclusion: There was no unexpected increased in mortality rate among patients undergoing cancer therapy during the COVID-19 pandemic as compared to the previous years of the same timeline. No increase in monthly mortality rates among patients receiving anti-cancer treatment were demonstrated during the pandemic period.
\end{abstract}

Keywords: COVID-19, Cancer, Mortality, Anticancer therapy, Pandemic.

How to cite this article: Demirci A, Bilir C, Cakir E, Gulbagci B, Hacibekiroglu I, Varim C. Has the COVID-19 Pandemic Increased Mortality Among Patients with Cancer Receiving Systemic Anticancer Treatments?. J Coll Physicians Surg Pak 2021; 31(JCPSPCR):CR66-CR70.

\section{INTRODUCTION}

Due to the coronavirus disease-19 (COVID-19) pandemic, additional measures have been taken in many countries for patients with cancer. In China, the first country to experience the outbreak, travel restrictions began on $23^{\text {rd }}$ January $2020 .^{1}$ After cases began to appear in Italy in the last week of February and showed a rapid increase, some restrictions wereestablished in all European countries during March. ${ }^{1,2}$ In Turkey, the Ministry of Health set up the Coronavirus Scientific Advisory Board on $10^{\text {th }}$ January 2020. The country's first coronavirus case was reported on March 10, 2020.

Correspondence to: Dr. Ayse Demirci, Department of Medical Oncology, Sakarya University Training and Research Hospital, Sakarya, Turkey

E-mail: aaysedemirci@gmail.com

Received: December 07, 2020; Revised: January 21, 2020;

Accepted: February 22, 2021

DOI: https://doi.org/10.29271/jcpsp.2021.JCPSPCR.CR66
Although patients with COVID-19 are especially vulnerable to lung disease, it also causes a wide range of infections that can affect the kidney, heart, central nervous system. ${ }^{3.5}$ Based on currently available information and clinical expertise, World Health Organization has stated that those whoareimmunocompromised, including those receiving cancer treatment, might be at higher risk for severe illness from COVID-19. It was easy to make decisions about patients who did not have active cancer or were in remission and whose consultation was made by phone call. However, this was more difficult when deciding about patients who had been diagnosed with new cancer or were already on chemotherapy.

In this study, the aim was to evaluate mortality rates in patients receiving anti-cancer therapy during the COVID-19 pandemic period.

\section{METHODOLOGY}

This was a single-centre, descriptive study. The files of all patients, who were followed-up and treated at Hospital Medical 
Oncology Clinic between 1 December 2017 and 31 May 2020, were examined retrospectively. Only patients who were receiving chemotherapy and immunotherapy were selected. Patients' age, gender, cancer type, presence of metastatic disease, type of chemotherapy, and treatment regimens were recorded from the files. The life status or date of death of the patients were collected from the hospital records. To better focus on the effects of the COVID-19 pandemic, only the first half of each year was included in the analysis.

All patients were divided into three groups, namely the first group (1st December 2017-31st May 2018, $n=918$ ), second group (1st December 2018-31st May 2019, $n=1,147$ ), and pandemic period group (PPG) (1st December 2019-31st May $2020, n=1,139$ ), according to the period when they received therapy. Descriptive statistics and mortality rates were compared between these groups, which reflect the first period of three consecutive years.

Non-palliative and palliative anti-cancer therapies included treatment options for the adjuvant, neoadjuvant, and metastatic stages. Patients who received chemotherapy and immunotherapy were described as patients receiving anticancer therapy. Anticancer treatments were classified into two different groups in terms of risk of febrile neutropenia ( $>20 \%$ high-risk, $<20 \%$ moderate / low-risk). ${ }^{6}$ Therapies consisting of single anticancer therapy and more than one drug were accepted as monotherapy and combination therapy, respectively.

All patients with solid malignancy over 18 years of age who were receiving active anticancer therapy were included in the study. Patients who did not receive anticancer therapy or who had only routine control, were excluded from the study. Since the patients with hematological malignancies and lymphoma were followed up by hematology, these patients were not included.

Data analysis was performed using SPSS-22 for Windows (Statistical Package for Social Science, SPSS Inc. Chicago IL, USA $® Z$ ). The variables were investigated using visual (histograms, probability plot) and analytical methods (Kolmogorov-Smirnov test) to determine whether or not they were normally distributed. Analyses were performed to describe and summarise the distributions of variables. Continuous variables were reported in terms of median and interquartile range (IQR). Categorical variables have been described in terms of frequencies and percentages. Kruskal-Wallis test was used to compare continuous nonparametric variables. The Chi-square test was used to compare the proportions in different groups. The statistically significant two-tailed $p$-value was set at $<0.05$.

\section{RESULTS}

A total of 3,204 cancer patients, who met the criteria, were evaluated. The median age of all patients was 61 (53-69). One thousand six hundred and thirty-six (51.1\%) patients were females and $48.9 \%(n=1,568)$ percent were males. Sixty-nine percent $(n=2,211)$ of all patients were at an advanced stage of cancer and, therefore, were receiving palliative chemotherapy. Most common cancer types were breast cancer $(25.9 \%$ [ $n=831])$, lung cancer $(23.8 \%[n=761])$, and colorectal cancer $(15.6 \%$ $[n=501])$, respectively. There were no differences between the median age and gender distribution of the patients who received anticancer therapy in the three groups included in the study $(p=0.555$ and $p=0.149$, respectively, Table I). The three most common types of cancer were lung, breast, and colorectal cancer in all three groups analysed. The distribution of these and other cancer types was not different between groups ( $p=0.061$ ). Additionally, metastatic disease rates were comparable between the groups. Metastatic disease rates were calculated as $67.9 \%$ in the first group, $73.3 \%$ in the second group, and $65.6 \%$ in the PPG, respectively $(p<0.001)$. While there was no significant difference between the first group and PPG in posthoc analysis (Bonferroni method), the second group had a statistically significantly higher ratethan the other groups.

The mortality rates were $13.5 \%(n=124)$ in the first group, $13.4 \%$ $(n=154)$ in the second group, and $13.0 \%(n=148)$ in the PPG. Overall mortality rates did not differ among the patients with cancer who received anticancer therapy in three different six-month periods analysed ( $p=0.931$, Figure 1 ). Monthly mortality changes were also evaluated and the results were different among the three groups $(p<0.001)$. The difference was due only to the months of April and May; while there was no difference between the second group $(2.4 \%[n=27])$ and PPG $(1.1 \%$ $[n=13])$. For April, the mortality rate was significantly higher in the first group (3.4\% [n=31]) than in the PPG. For May, the PPG mortality rate $(0.8 \%[n=9])$ was lower than both the first group $(3.4 \%[n=31])$ and the second group (3.3\% $[n=38])$. These findings are summarised in Figure 2 . The median ages of the patients who died were 66 (58-71), 65 (58-72), and 65 (57-70), respectively; and the difference was not significant $(p=0.560)$. In addition, while the commonest histological type observed in patients who died in all three groups was lung cancer $(47.6 \%$ [ $n=59$ ], 38.3\% [ $n=59$ ], 37.8\% [ $=56]$, respectively). The distribution of cancer types did not significantly differ between groups $(p=0.690)$.

In the PPG, six of nine COVID-19 positive (confirmed real-time polymerase chain reaction [RT PCR] from nose or throat swab) patients died who had received chemotherapy within four weeks before testing positive for COVID-19. The COVID-19 positive death rate was $4 \%$ in 148 patients who died in total. Four of these six patients had metastatic lung cancer, and two had colorectal cancer. They were all older than 55 years. Only one patient had received curative chemotherapy.

Comparing the febrile neutropenia risk of regimens, there were no differences in mortality rates over the years $(p=0.157$ for $>$ $20 \%$ and $p=0.632$ for $<20 \%$ ). Moreover, there was no significant difference between the groups when compared according to the purpose of administration as palliative orcurative $(p=0.661$ and $p=0.776$, respectively). Finally, no significant changes in mortality rates were detected within three years with regard to monotherapy or combination therapy $(p=0.375$ and $p=0.731$, respectively). Thesefindings are summarized in Figure 3. 
Table I: Comparison of the baseline characteristics of the groups.

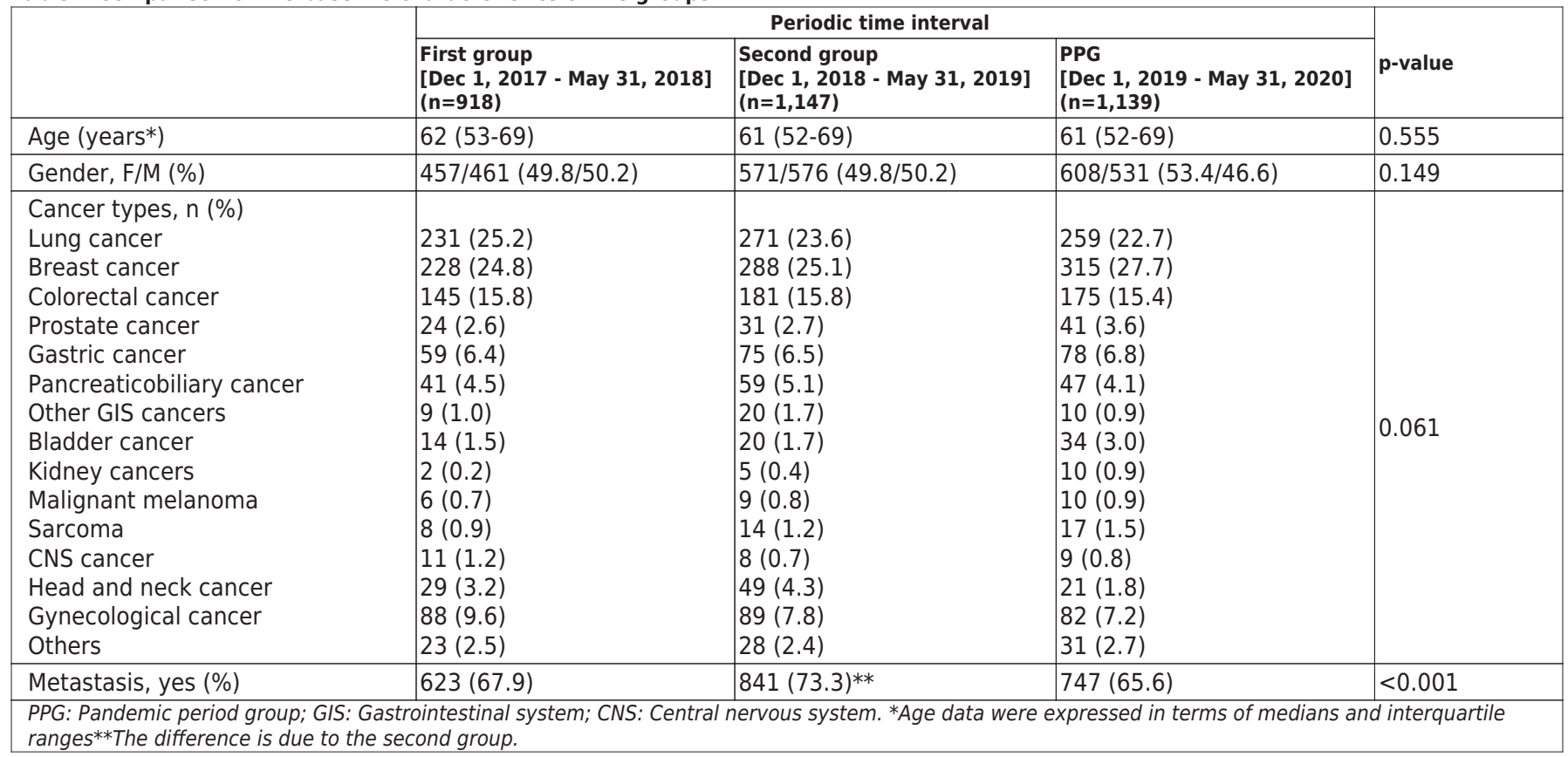

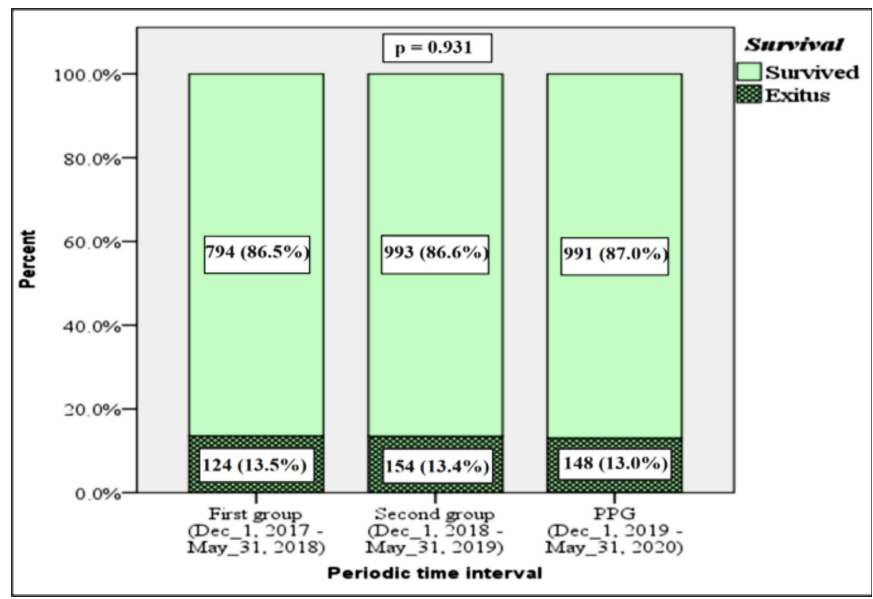

Figure 1: Overall mortality rates over three different time periods (PPG = Pandemic period group).

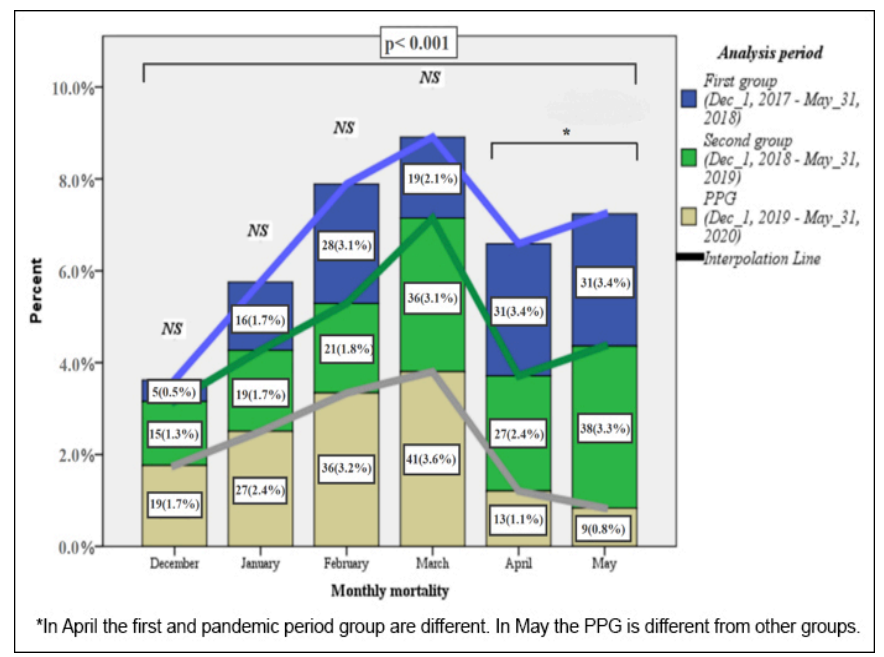

Figure 2: Comparison of mortality by months in annual periods.

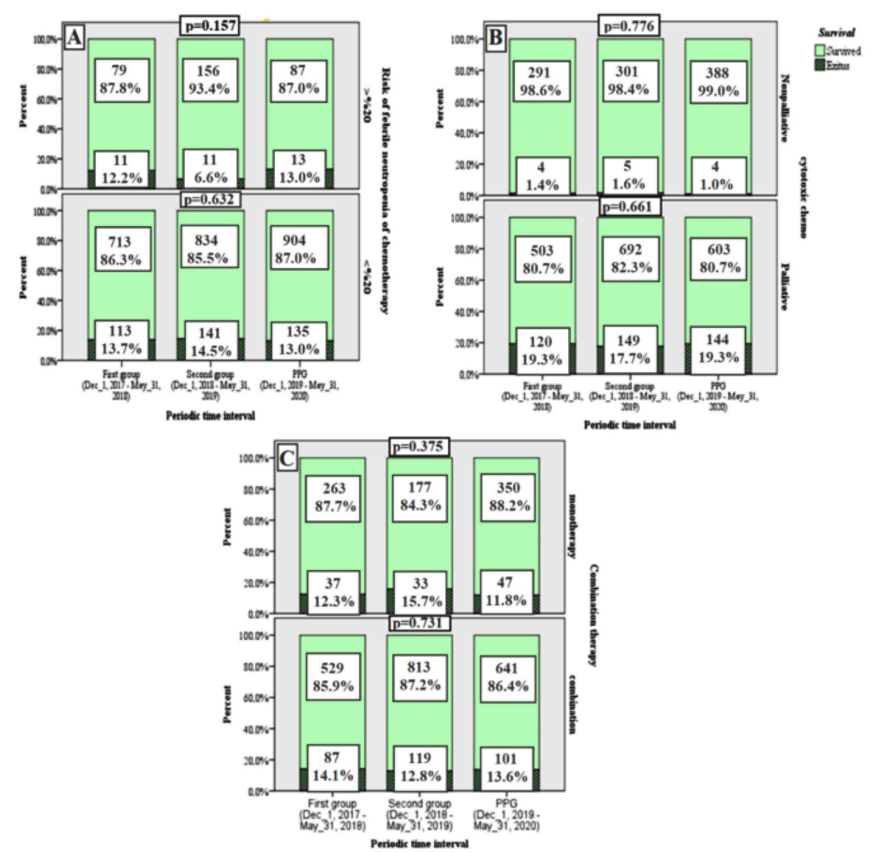

Figure 3. Mortality results according to chemotherapy options. Comparison according to the risk of febrile neutropenia (A), whether it is given for palliative purposes (B) and whether it is applied in combination (C). PPG = Pandemic period group.

\section{DISCUSSION}

There was no unexpected higher mortality rate of cancer therapy during COVID-19 pandemic as compared to the previous years of the same timeline. Overall obtained results showed that there was no increase in mortality among patients with cancer receiving anticancer therapy, which was accepted as in high-risk group during the COVID-19 pandemic period, as compared to the 2018 and 2019 seasons. 
Following the first COVID-19 case seen in Turkey, it was tried to reduce the patient value by delaying control examinations among outpatients without active cancer. After some prevention to reduce the number of patients, an average of 50 patients in an outpatient setting were evaluated. These numbers were about half of the patients who were examined in pre-pandemic conditions. The number of patients receiving chemotherapy did not change during this period. Patients with cancer are more susceptible to infectious agents and infection-related deaths than other healthy individuals due to immunosuppression. Although there have been studies on the rates of cancer patients among those who had COVID-19 in the whole population and who died due to COVID-19, no further studies are available on the effect of the mortality rate of patients with cancer receiving anticancer treatment. The first data on this issue was reported from China, where the pandemic first appeared. Liang et al. reported the results of a prospective cohort. ${ }^{7}$ The results of 1,590 COVID-19 cases with sufficient data were evaluated, and 18 patients had a history of cancer. While cancer incidence in the Chinese population was $0.29 \%$, they found a higher cancer incidence in COVID-19 cases, which was $1 \%$. In this study, the authors defined serious events requiring mechanical ventilation in the intensive care unit, and the death rate was higher in cancer patients than in patients without cancer (38.9 [7 of 18 patients] and 6.6 [124 of 1,872 patients], $p<0.001$, respectively). However, the proportion of patients with cancer among all deaths was not specified separately. It was also found that patients who had undergone chemotherapy or surgery last month had a numerically higher risk $(43.0 \%$ vs. $75.0 \%)$ of clinically serious events compared to those who did not receive chemotherapy or surgery. According to the results of this study, patients with cancer have been shown to have a higher risk of COVID-19 than other individuals. COVID-19 positive patients with cancer have been shown to have worse results because they are immunosuppressed. ${ }^{7}$ According to the statistics of the Turkish National Ministry of Health, between March 11 and May 31, 2020, 2,074,794 people were screened for COVID-19, and 164,781 were found positive $(7.9 \%)$. Between these dates, the general population mortality rate was declared as $2.77 \%$. However, in the same period, the total number of patients receiving anticancer treatment in this centre was 835, and 9 of these patients were symptomatic and documented as COVID-19 positive. $^{8}$

In another study from the United States of America, which had a rapid increase in the number of cases in March, the rate of cancer patients among 5,688 COVID-19 positive patients between March 1 and April 6 was determined as $6 \%$ $(n=334) .{ }^{9}$ The risk of intubation was higher in cancer patients without adjustment for age groups (relative risk [RR]; 1.89 [1.37-2.61]), but the mortality rate was not significantly different. Although the risk of intubation was found to be significantly higher in cancer patients aged
66-80 years, the risk of death at this age was not significantly higher. Moreover, the risk of death was found to be significantly higher (RR [95\% confidence interval]; 5.01 [1.55 - 16.2]) in patients $<50$ years of age with cancer. ${ }^{9}$

In the multicenter study by Dai et al., 107 COVID-19 positive patients with cancer and 536 noncancer patients were compared. In patients with COVID-19 positive cancer, the serious event incidence was higher especially in hematologic cancer, lung cancer, and metastatic cancer. ${ }^{10}$

Lee et al. published a prospective multicenter observational study to describe COVID-19 outcomes in patients with

cancer. ${ }^{11}$ Eight hundred patients diagnosed with cancer and symptomatic COVID-19 (confirmed by RT PCR from nose or throat swab) were analysed, in which $226(28.3 \%)$ patients died. The number of patients who received cytotoxic chemotherapy within 4 weeks before the positive test for COVID-19, was 281 (35.1\%). When the characteristics of patients with cancer who died were examined, it was concluded that mortality rates were not different between patients who received cytotoxic chemotherapy in the last four weeks and those who did not. In agreement with that study, the present study displayed the absence of an increase in mortality in the period corresponding to the COVID-19 pandemic in patients who received anticancer treatment compared to other years.

In this study, the higher mortality in December, January, February, and March compared to April and May may be because the overall mortality is higher in cooler than in warmer months in Europe, and non-tropical Asian cities have reported increased mortality in winter. ${ }^{12,13}$ Likewise, in a study from Hungary, which has similar climatic conditions as this study centre, significant winter peak seasonality was shown in mortality rates from all cancers. ${ }^{14}$

The strength of this study was the high number of patients and similar distribution in terms of the subtype of cancer, age, and gender among the three groups. This data were obtained from patient records in a robotic drug preparation system, which is currently used to prepare parenteral anticancer treatments in this hospital.

The major limitation of the study was that the authors did not confidently exclude COVID-19 diagnosis in all deceased patients but, the patient population was homogenous with regard to previous years as age, gender, cancer type, presence of metastatic disease, type of chemotherapy, and application purpose. Additionally, hospital records and phone call surveys excluded the COVID-19 diagnosis and possible viral contamination of the patients.

Another limitation of the study was that the authors did not enroll patients treated with other than cytotoxic drugs. As a result of precautions taken during the pandemic period, patients who received oral anti-cancer treatment were not 
included because the frequency of visits to the hospital decreased. They could obtain their medicines from pharmacies post-follow-up and continue with telemedicine.

Further limitation of this study is that it did not include hematological cancers. On the other hand, there was no increased mortality rate of lung cancer and metastatic cancer compared to previous years, as most of the patients who died had metastatic disease.

\section{CONCLUSION}

Due to the susceptibility to infection in immunosuppressive patients receiving anticancer therapy, COVID-19 may be a primary potential causative agent, but this pandemic did not significantly change mortality rates in this group of patients as compared to previous years. These results showed that anticancer treatments during the COVID-19 pandemic period can safely be carried out.

\section{ETHICAL APPROVAL:}

Ethics committee approval was obtained from the Ministry of Health of the Turkish Republic and the Sakarya University Medical School (No: 16214662/050.01.04; April 13, 2020).

\section{PATIENTS' CONSENT:}

Informed consents have been obtained from all subjects.

\section{CONFLICT OF INTEREST:}

Authors declared no conflict of interest.

\section{AUTHORS' CONTRIBUTION:}

AD: Data curation, planning the study design, writing original draft preparation, reviewing and editing.

CB: Data collecting, quality control of data and algorithms, writing original draft preparation.

EC: Contributed in statistical data analysis, interpretation, collection and content writing.

BG: Contributed in data collection and interpretation.

$\mathrm{IH}$ : Participated in data interpretation and supervised the intellectual contents.

CV: Data collecting, quality control of data and participated in statistical analysis of data.

\section{REFERENCES}

1. Oksanen A, Kaakinen M, Latikka R, Savolainen I, Savela N, Koivuia A. Regulation and Trust: 3-Month Follow-up Study on COVID-19 Mortality in 25 European Countries. JMIR Public Heal Surveill2020; 24(6):e19218. doi: 10.2196/ 19218
2. Remuzzi A, Remuzzi G. COVID-19 and Italy: what next? Lancet 2020; 395(10231):1225-8. doi: 10.1016/S01406736(20)30627-9.

3. Asgharpour M, Zare E, Mubarak M, Alirezaei A. COVID-19 and Kidney Disease: Update on Epidemiology, Clinical Manifestations, Pathophysiology and Management. J Coll Physicians Surg Pak 2020; 30(6):19-25. doi: 10.29271/ jcpsp. 2020.Supp1.S19.

4. Losy J. SARS-CoV-2 Infection: Symptoms of the Nervous System and Implications for Therapy in Neurological Disorders. Neurol Ther 2020; 23:1-12. doi: 10.1007/ s40120-020-00225-0.

5. Magadum A, KishoreR. Cardiovascular Manifestations of COVID-19 Infection. Cells 2020; 9(11):2508. doi: 10.3390/ cells9112508.

6. National Comprehensive Cancer Network. Hematopoietic Growth Factors, version 2.2020-January 27,2020 http:// www.nccn.org/professionals/physician_gls/pdf/growthfactor s.pdf (2020, accessed 18 August 2020).

7. Liang W, Guan W, Chen R, Wang W, Li J, Xuet K, et al. Cancer patients in SARS-CoV-2 infection: a nationwide analysis in China. Lancet Oncol 2020; 21(3):335-37. doi: 10.1016/S1470-2045(20)30096-6.

8. Republic of Turkey Ministry of Health. COVID-19 Situation Report. http://covid19.saglik.gov.tr/ (2020, accessed 18 August 2020).

9. Miyashita H, Mikami T, Chopra N, Yamada T, Chernyavsky S, RizkD, et al. Do patients with cancer have a poorer prognosis of COVID-19? An experience in New York City. Ann Oncol 2020; 31(8):1088-89. doi: 10.1016/j.annonc. 2020.04.006.

10. Dai M, Liu D, Liu M, Zhou F, Li G, Chen Z, et al. Patients with cancer appear more vulnerable to SARS-CoV-2: A multicenter study during the COVID-19 outbreak. Cancer Discov 2020; 10(6):783-91. doi: 10.1158/2159-8290. CD-20-0422.

11. Lee LYW, Cazier JB, Angelis V, Arnold R, Bisht V, Campton NA, et al. COVID-19 mortality in patients with cancer on chemotherapy or other anticancer treatments: A prospective cohort study. Lancet 2020; 395(10241): 1919-26. doi: 10.1016/S0140-6736(20)31173-9.

12. Healy JD. Excess winter mortality in Europe: A cross country analysis identifying key risk factors. J Epidemiol Community Health 2003; 57(10):784-89. doi: 10.1136/ jech.57.10.784.

13. Goggins WB, Chan EYY, Yang C, Goggins MC. Associations between mortality and meteorological and pollutant variables during the cool season in two Asian cities with subtropical climates: Hong Kong and Taipei. Environ Health2013; 19:1-10. doi: 10.1186/1476-069X-12-59.

14. Virág K. Nyári TA. Seasonal variation of cancer mortality in Hungary between 1984 and 2013. Scand. J. Public Health 2019; 47(5):492-96. doi: 10.1177/1403494817744074. 